\title{
FORUM
}

\section{Enabling HIV self-testing in South Africa}

M L Richter, W D F Venter, A Gray

African Centre for Migration and Society, University of the Witwatersrand, Johannesburg, and International Centre for Reproductive Health, Department of Obstetrics and Gynecology, Ghent University, Belgium

M L Richter, BA (Hons) MA LLM

Wits Reproductive Health and HIV Institute, University of the Witwatersrand, Johannesburg

W D F Venter, FCP (SA)

Discipline of Pharmaceutical Sciences, University of KwaZulu-Natal, Durban

A Gray, MSc (Pharm), FPS

Corresponding author: M L Richter (marlise.richter@gmail.com)

In a South African context, we consider the implications of the United States Food and Drug Administration's recent approval of the OraQuick HIV self-testing kit. We argue that current law and policy inhibit the roll-out of accurate and well-regulated self-testing kits, and create a loophole for sale in supermarkets, but not pharmacies.

S Afr J HIV Med 2012;13(4):186-187. DOI:10.7196/SAJHIVMED.858

In July 2012, the Food and Drug Administration (FDA) approved the OraQuick test (OraSure Technologies) as the first HIV self-testing kit in the USA. ${ }^{1}$ This over-the-counter test, retailing at approximately US\$35 - 40 (R245 - 280), uses saliva from a mouth swab and provides a result within 20 40 mins. OraSure has established a consumer support centre that provides telephonic support and referrals. ${ }^{2}$ In a recent study of New York-based men who have sex with men (MSM) who were provided with the self-testing kit, few experienced problems performing the test. ${ }^{3}$ Studies by the Integration of TB in Education and Care for HIV/AIDS (iTEACH) in rural KwaZulu-Natal, South Africa (SA), are showing equally promising results (K Dong, personal communication). It would seem that, with appropriate support, self-testing is poised to revolutionise HIV-testing.

SA has made immense strides in improving HIV testing coverage, at least partly owing to direct intervention by the Minister of Health. ${ }^{4}$ However, average CD4 counts at initiation of HIV treatment remain low, suggesting that late diagnosis may still be a problem for a sizeable proportion of the population. New, more convenient ways to test for HIV may increase the proportion of individuals who know their HIV status, and help to identify infected individuals earlier in the course of disease. A recent Lancet editorial notes: 'Ironically, the lack of mandatory counselling with OraQuick may help decrease the stigma around testing.'

Previously, we examined the arguments against self-testing and showed that critics' objections to its roll-out in SA were based largely on vague fears with little supporting evidence. ${ }^{6}$ Similar conclusions in support of self-testing have been drawn by others. ${ }^{7.8}$ There are a wide range of self-tests currently available in pharmacies and supermarkets in SA, including tests for pregnancy, prostate cancer, ovulation, recreational drugs and breathalysers for alcohol. Few objections have been raised against the availability of these tests, and their distribution is not regulated. We have argued that self-testing in SA may have an enormous positive effect on HIV testing uptake and early diagnosis. Self-testing could extend to groups that have been traditionally hard to reach with general public health campaigns, and would be in line with the spirit of the Patients' Rights Charter and the National Health Act, urging people to take responsibility for their own health. ${ }^{6}$

Yet, SA's legal and policy frameworks do not facilitate the dissemination of HIV self-tests. Self-tests are classified as 'medical devices' under the Medicines and Related Substances Control Act (Act no. 101 of 1965, as amended), but there is no regulatory system in place yet for medical devices. This means, for example, that the manufacturers of the OraQuick test would be able to market it in SA - as long as the kit, ironically, is not available in pharmacies. The only legally binding restriction on the distribution of selftesting HIV kits is provided by the Good Pharmacy Practice (GPP) standards issued by the South African Pharmacy Council. ${ }^{9}$ The 4th edition of the GPP, last updated in 2010, prevents pharmacists from selling the test or administering it in a pharmacy. Section 2.13.5.5 of the GPP states that 'only rapid tests which use a blood sample may be performed in a pharmacy'. Section 2.13.5.8(h) adds that 'pharmacists must not sell HIV tests for patients to perform at home.' Interestingly, this restriction does not apply to any other tests. Nor does the GPP apply to general supermarkets or corner cafes, creating a loophole for distribution. While the 
FDA stamp of approval means that the public need not be concerned about the accuracy of OraQuick if it were to become available locally, this would not necessarily hold true for other HIV self-tests that are currently obtainable at community pharmacies, and are left unregulated. Although implementing an effective regulatory system for medical devices is challenging, unjustified restrictions such as those in the GPP could easily be addressed.

A recent Civil Society Consensus Statement on strategies to improve HIV testing and counselling highlighted these and other challenges of HIV-testing policies, paradigms and legal frameworks in SA. ${ }^{10}$ The Statement endorsed self-testing if accompanied by the same essential components of any HIV testing service, including easy access to accurate information' and linkages to care. These are indeed vital components of the goal to enable everyone in SA to test for HIV regularly, and to do so when and where they choose.

\section{Useful websites:}

- 'The first in-home oral HIV test': http:// www.oraquick.com/home

- 'OraQuick In-Home HIV Test': http://www. cvs.com/shop/product-detail/OraQuickin-home-HIV-test?skuId=896631

References

1. British Broadcasting Corporation (BBC) News. US approves first over-the-counter HIV home-use test. London: BBC News, 2012. http://www.bbc.co.uk/news/ world-us-canada-18700121 (accessed 3 July 2012).

2. Food and Drug Administration (FDA). First Rapid Home-Use HIV Kit Approved for Self-Testing. Silver Spring, MD: FDA, 2012. http://www.fda.gov/ ForConsumers/ConsumerUpdates/ucm 310545.htm (accessed 24 October 2012).

3. Carballo-Diéguez A, Frasca T, Balan I, Ibitoye M, Dolezal C. Use of a rapid HIV home test prevents HIV exposure in a high risk sample of men who have sex with men. AIDS and Behavior 2012;16(7):1753-1760. [http://dx.doi.org/10.1007/ s10461-012-0274-2

4. Mbengashe T. The National HIV Counselling and Testing Campaign and Treatment Expansion in South Africa: A Return on Investments in Combination Prevention. XIX International AIDS Conference, 26 July 2012, Washington DC.

5. Arnold C. At-home HIV test poses dilemmas and opportunities. Lancet 2012;380(9847):1045-1046.

6. Richter M, Venter WD, Gray A. Home self-testing for HIV: AIDS exceptionalism gone wrong. S Afr Med J 2010;100(10):636-642.

7. Gardner J. HIV home testing - a problem or part of the solution? South African Journal of Bioethics and Law 2012;5(1):15-19.

8. Bayer R, Stryker J, Smith MD. Testing for HIV infection at home. N Engl J Med 1995;332(19):12961299.

9. South African Pharmacy Council. Good Pharmacy Practice in South Africa. Fourth Edition. Arcadia: South African Pharmacy Council, 2010.

10. Southern African HIV Clinicians Society, Treatment Action Campaign, SECTION27, et al. Joint Statement - How We Can Improve HIV Testing and Counselling. http://www.sahivsoc.org/ newsroom/society-news (accessed 30 July 2012). 